\title{
Dissociation of Water on Defective Carbon Substrates
}

\author{
M. K. Kostov, ${ }^{1, *}$ E. E. Santiso, ${ }^{1, *}$ A. M. George, ${ }^{2}$ K. E. Gubbins, ${ }^{1}$ and M. Buongiorno Nardelli ${ }^{2,3, \dagger}$ \\ ${ }^{1}$ Center for High Performance Simulation and Department of Chemical and Biomolecular Engineering, North Carolina State \\ University, Raleigh, North Carolina 27695, USA \\ ${ }^{2}$ Center for High Performance Simulation and Department of Physics, North Carolina State University, \\ Raleigh, North Carolina 27695, USA \\ ${ }^{3}$ CCS-CSM, Oak Ridge National Laboratory, Oak Ridge, Tennessee 37831-6359, USA
}

(Received 2 June 2005; published 23 September 2005)

\begin{abstract}
Using calculations from first principles, we found that water can dissociate over defective sites in graphene or nanotubes following many possible reaction pathways, some of which have activation barriers lower than half the value for the dissociation of bulk water. This reduction is caused by spin selection rules that allow the system to remain on the same spin surface throughout the reaction.
\end{abstract}

DOI: 10.1103/PhysRevLett.95.136105

The direct thermal splitting of water, $\mathrm{H}_{2} \mathrm{O} \rightarrow \mathrm{H}_{2}+\frac{1}{2} \mathrm{O}_{2}$, which does not generate $\mathrm{CO}_{2}$ and produces highly pure hydrogen, requires temperatures in excess of $2000^{\circ} \mathrm{C}$ thus making its practical implementation very challenging [1]. In addition, it has a relatively low hydrogen yield of about $25 \%$ [2]. The fundamental limitation of the thermal splitting of bulk water is the fact that the ground state of oxygen is paramagnetic (a spin triplet), whereas the ground state of water is diamagnetic (a spin singlet). Breaking a water molecule thus requires an excess of $45 \mathrm{kcal} / \mathrm{mol}$ of additional energy to excite the molecule to a higher spin state prior to the dissociation. This energy is later released as unrecovered heat when oxygen relaxes to its ground state. In this work, we propose an alternative paradigm in $\mathrm{H}_{2}$ production: a process in which the system remains on the singlet spin surface throughout the reaction by exploiting the reactivity of defects in carbon materials.

Graphitic carbon substrates such as activated carbon fibers, nanotubes, and fullerenes have been extensively studied for their potential as media for energy storage and $\mathrm{H}_{2}$ sequestration [3-5]. However, there is no report of using them as chemical reactors rather than fuel tanks. Using ab initio density functional theory [6,7] (DFT) techniques, we have found evidence that if structural defects are present or are artificially created in graphiticlike materials, a rich chemistry emerges, yielding many possible water dissociation pathways to produce hydrogen, some of which have activation barriers lower than half the value for the dissociation of bulk water. This means that the production of hydrogen can be achieved at temperatures below $1000^{\circ} \mathrm{C}$. In particular, in Fig. 1 we show the estimated equilibrium yield for the dissociation of water on a single vacancy in a nanotube and a graphene sheet as compared with the direct thermolysis of the water molecule in free space. It is clear that the defective carbon substrate can produce hydrogen from water at temperatures at least a factor of 2 lower for hydrogen yields comparable to the free space reaction.
PACS numbers: 82.65.+r, 68.43.Bc, 82.20.Rp, 82.30.Lp

Recent studies have found experimental evidence for the existence of a variety of metastable defects in graphitic carbon materials [8]. These defects can be as grown or can be induced in both graphene layers and carbon nanotubes by employing electron or ion irradiation $[9,10]$. Among these defects, monovacancies, in particular, are the most common and they have been experimentally shown to be numerous and stable. Materials containing such defects have potential applications as catalysts, due to the presence of carbon atoms with unsaturated valence shells in their structures.

The electronic structure calculations presented in this work were done using plane-wave density functional theory (DFT) [11] with ultrasoft pseudopotentials [12] as implemented in the CPMD [13] and PWSCF [14] codes. We considered both the Becke-Lee-Yang-Parr $[15,16]$ (BLYP) and Perdew-Becke-Ernzerhof [17] (PBE) exchangecorrelation functionals and found negligible differences in our final results. For graphene we used a periodic super-

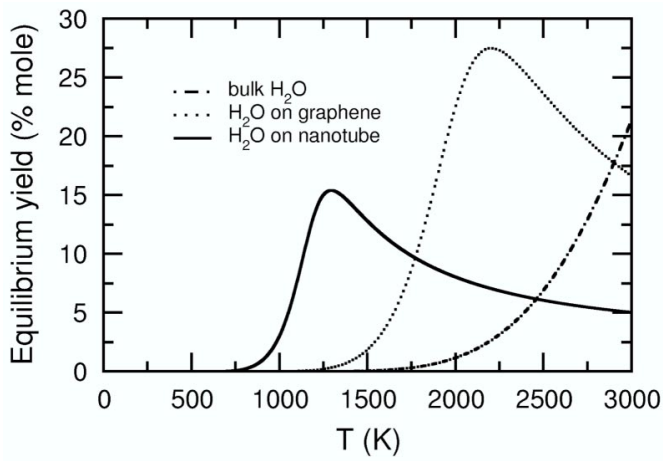

FIG. 1 (color online). Ideal-gas equilibrium yields (fraction of the water that is converted to hydrogen at equilibrium in \% mole) for the dissociation of water in bulk, over a single vacancy in graphene, and over a single vacancy in a 10-10 carbon nanotube. The values correspond to a pressure of 1 bar and an initial equimolecular mixture of water and vacancies. See text for a more detailed discussion. 
cell with 31 carbon atoms (see Fig. 2), while we simulated the curved nanotube shell as a finite section of 39 carbon atoms with edges saturated with hydrogen (see Fig. 4, H atoms not shown in the picture).

The study of the water splitting on the vacancy requires the knowledge of all possible stable states, transition states, and reaction pathways, and the potential energy surface (PES) for this system is quite complicated due to the presence of several "dangling bonds." For this reason, the exploration of the PES has been done using a combination of techniques. First, we identified stable intermediates using the Laio-Parrinello metadynamics $[18,19]$ method. The configurations of all the energy minima obtained from the metadynamics were further relaxed with a strict convergence criterion in both singlet $(S=0)$ and triplet $(S=1)$ conformations.

Reaction pathways were obtained using the nudged elastic band [20,21] (NEB) method of Jónsson et al. with the climbing image modification [22]. In some cases the structure of the transition state was refined using the rational function optimization (RFO) method [23]. As a test for the accuracy of our approach we have verified that our data for the splitting of a single bulk water molecule agreed with experimental results and other theoretical calculations. Finally, the standard Gibbs free energy changes for all reactions were computed from the molecular partition functions [7] and used to obtain the equilibrium yields at different temperatures in the ideal-gas limit.

Vacancies in carbon nanomaterials indeed have the potential to dissociate water: their formation energy in graphene is approximately $\sim 170 \pm 10 \mathrm{kcal} / \mathrm{mol}$ [24,25] whereas the reaction enthalpy for the splitting of water is $116 \mathrm{kcal} / \mathrm{mol}$ [26]. The ground state shows a paramagnetic

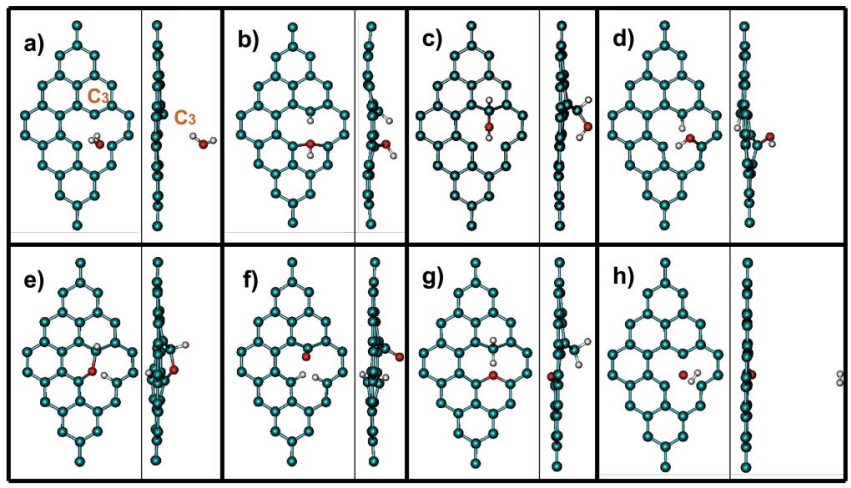

FIG. 2 (color online). Top and side views of various intermediate states in the water splitting reaction over a vacancy site in graphene. (a) Initial physisorbed state; (b) IM1: spin-singlet state with the highest energy; (c) IM2: spin-singlet state; (d) IM3: the only spin-triplet state $(S=1)$; (e) IM4: spin-singlet state; (f) $K$ (for "ketone"): spin-singlet state; (g) $E$ (for "ether"): spin-singlet state. This is the global minimum of the potential energy surface; (h) $F$ (for "final"): spin-singlet state; the oxygen occupies the vacancy site on the surface and hydrogen is away from it. structure where the $D_{3 h}$ symmetric vacancy undergoes a Jahn-Teller distortion, leading to out-of-plane motion of one of the "dangling bond" atoms [which we denote by $\mathrm{C}_{3}$; see Fig. 2(a)] [24].

In the full exploration of the PES, shown in Fig. 3, we chose the relaxed physisorbed water molecule over the center of the vacancy as the starting ("reactant") point of the reaction and used it as the energy reference in all calculations. We labeled this state by $I$ [for "initial," see Fig. 2(a)]. In addition, we found seven other stable structures that are relevant for the decomposition reaction, including the final state $F$ ["final," Fig. 2(h)] where the hydrogen molecule is fully separated. All the stable structures, including the $I$ and $F$ states, are shown in Fig. 2.

From this knowledge, we investigated all the possible reaction pathways leading to the final state $F$. Figure 3 shows the full PES for the reaction while the corresponding energy barriers $E_{A}$ are reported in Table I. This complex energy surface exhibits several favorable reaction channels for producing hydrogen. All these reaction pathways are isogyric, i.e., the system stays on the spin-singlet surface, which lowers the reaction barrier for dissociation. Among those favorable channels are: $I \rightarrow I M 1 \rightarrow F$ (with maximum energy barrier $\left.E_{A, \max }=16 \mathrm{kcal} / \mathrm{mol}\right), I \rightarrow K \rightarrow F$ $\left(E_{A, \max }=49 \mathrm{kcal} / \mathrm{mol}\right), \quad I \rightarrow I M 2 \rightarrow F \quad\left(E_{A, \max }=\right.$ $58 \mathrm{kcal} / \mathrm{mol}$ ), and the "straight dissociation" $I \rightarrow F$ $\left(E_{A, \max }=55 \mathrm{kcal} / \mathrm{mol}\right)$. In the straight dissociation reaction we observe a "roaming atom" reaction mechanismone hydrogen atom explores a large region of the potential energy surface before binding with the second $\mathrm{H}$ atom. Such a reaction mechanism has been just recently discov-

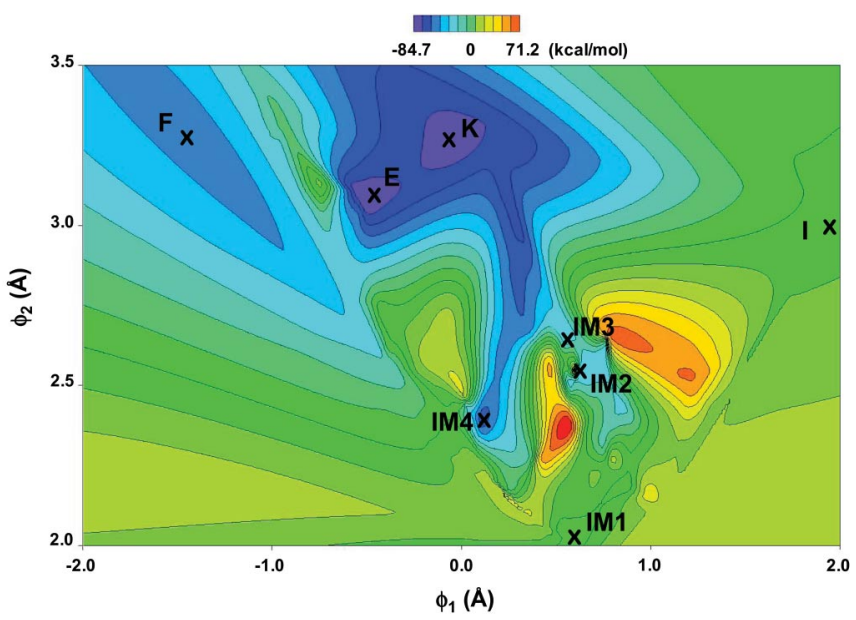

FIG. 3 (color online). Contour plot of the PES for the water dissociation reaction on a vacancy in graphene (units are in $\mathrm{kcal} / \mathrm{mol}$ ), as obtained by interpolation of the NEB trajectories. Different configurations are labeled as in Fig. 2. The order parameters $\phi_{1}$ and $\phi_{2}$ are defined as $\phi_{1}=(\langle\mathrm{CO}\rangle-$ $\langle\mathrm{OH}\rangle) / \sqrt{2})$ and $\left.\phi_{2}=(\langle\mathrm{CO}\rangle+\langle\mathrm{OH}\rangle) \sqrt{2}\right)$, where $\langle\mathrm{CO}\rangle$ is the average distance between the oxygen atom and the carbons immediately adjacent to the vacancy, and $\langle\mathrm{OH}\rangle$ is the average distance between the oxygen atom and the hydrogen atoms. 


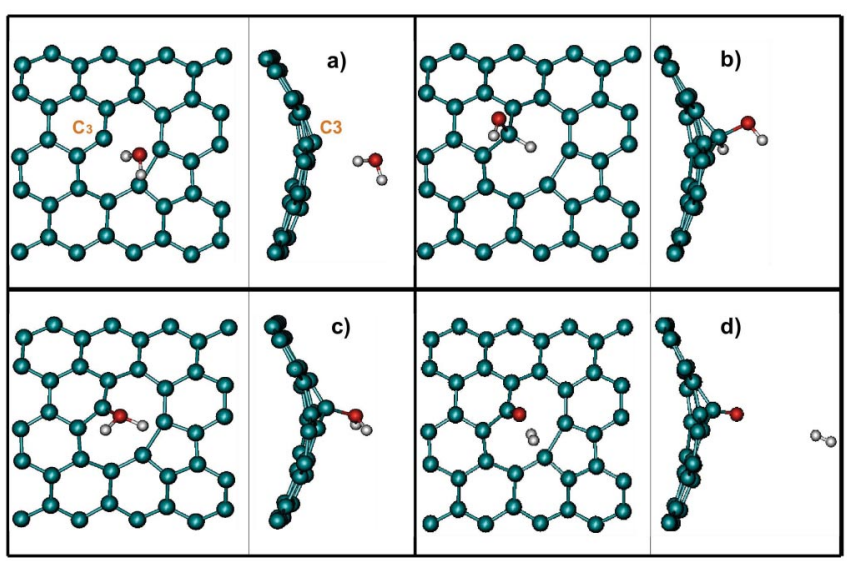

FIG. 4 (color online). Top and side views of (a) the initial $(N-I)$, (b) intermediate $(N-I M 2)$, (c) transition state $(N-T S T)$, and (d) final $(N-F)$ conformations for a favorable dissociation pathway over a vacancy in a $(10,10)$ nanotube.

ered in the $\mathrm{HCHO}$ decomposition reaction [27]. In reactions involving intermediate states, the process exploits different mechanisms, depending on the species that saturate the $\mathrm{C}_{3}$ "dangling bond" first. In the pathways through $K$ and $I M 4$ states, the $\mathrm{O}$ atom saturates $\mathrm{C}_{3}$ with a very small barrier $(3 \mathrm{kcal} / \mathrm{mol})$, whereas going through $I M 1$ and $I M 3$ involves the saturation of $\mathrm{C}_{3}$ by $\mathrm{H}$, still with very low energy barriers (16 and $15 \mathrm{kcal} / \mathrm{mol})$. In $I M 2$, where both $\mathrm{OH}$ and $\mathrm{H}$ are bonded with $\mathrm{C}_{3}$, the barrier is relatively larger $(33 \mathrm{kcal} / \mathrm{mol})$ but still small compared to the straight dissociation. The number of possible reaction pathways that branch from these intermediate states is large and is best summarized by the activation energy data reported in Table I. An analysis of the data in this table and in Fig. 3 reveals that, at moderate temperatures, the most favorable pathway to the $F$ state is $I \rightarrow I M 1 \rightarrow$ $F$, and the most important competing reactions are the ones toward the $K$ and $E$ states. This is consistent with the equilibrium data shown in Fig. 1, where it is seen that at low temperatures, the formation of $E$ and $K$ are most favorable, with the equilibrium shifting towards $F$ at moderate temperatures. None of the other intermediate states are appreciably present.

With the goal of further increasing the efficiency of the dissociation process and the selectivity towards the final dissociated state, we investigated whether the curvature of the reactive substrate, as in a nanotube, could improve upon the reaction mechanism.

In general, the removal of any $\mathrm{C}$ atom in a hexagonal graphite network implies the creation of three twocoordinated $\mathrm{C}$ atoms. When the atomic layer is strongly curved, however, it is possible for two of the "dangling bond" atoms to recombine, thus creating a pentagon. This structure is more stable, since the reconstructed bond is much shorter compared to the distance between two "dangling bond" atoms in graphene $[1.65 \AA$ for a $(10,10)$ tube versus $2.46 \AA$ for graphene]. This different conformation
TABLE I. Activation energies for the forward $\left(E_{A, f}\right)$ and backward $\left(E_{A, r}\right)$ reactions and total energy change $(\Delta E)$ for each of the reaction steps in planar graphene.

\begin{tabular}{|c|c|c|c|}
\hline Reaction & $E_{A, f}(\mathrm{kcal} / \mathrm{mol})$ & $E_{A, r}(\mathrm{kcal} / \mathrm{mol})$ & $\Delta E(\mathrm{kcal} / \mathrm{mol})$ \\
\hline Bulk & 131 & 16 & 115 \\
\hline$I \rightarrow I M 1$ & 16 & 24 & -8 \\
\hline$I \rightarrow I M 2$ & 33 & 44 & -11 \\
\hline$I \rightarrow I M 3$ & 15 & 56 & -41 \\
\hline$I \rightarrow I M 4$ & 3 & 68 & -65 \\
\hline$I \rightarrow K$ & 3 & 79 & -76 \\
\hline$I M 1 \rightarrow F$ & 16 & 47 & -31 \\
\hline$I M 1 \rightarrow I M 2$ & 28 & 31 & -3 \\
\hline$I M 1 \rightarrow I M 3$ & 4 & 37 & -33 \\
\hline$I M 1 \rightarrow E$ & 28 & 107 & -79 \\
\hline$I M 1 \rightarrow K$ & 3 & 71 & -68 \\
\hline$I M 2 \rightarrow F$ & 58 & 85 & -27 \\
\hline$I M 2 \rightarrow K$ & 21 & 86 & -65 \\
\hline$I M 2 \rightarrow E$ & 38 & 114 & -76 \\
\hline$I M 3 \rightarrow I M 2$ & 55 & 25 & 30 \\
\hline$I M 3 \rightarrow K$ & 14 & 49 & -35 \\
\hline$I M 3 \rightarrow F$ & 112 & 110 & 2 \\
\hline$I M 3 \rightarrow E$ & 60 & 106 & -46 \\
\hline$I M 4 \rightarrow F$ & 80 & 53 & 27 \\
\hline$I M 4 \rightarrow K$ & 13 & 24 & -11 \\
\hline$K \rightarrow E$ & 72 & 83 & -11 \\
\hline$K \rightarrow F$ & 49 & 12 & 37 \\
\hline$E \rightarrow F$ & 94 & 46 & 48 \\
\hline Straight & 55 & 94 & -39 \\
\hline
\end{tabular}

makes the system less reactive, since now the water molecule can saturate readily only one "dangling bond."

We have identified several stable intermediate states inspired by the analogy with the planar graphene case and found important differences in the reaction mechanisms solely induced by curvature effects. In the initial physisorbed state $(N-I)$, shown in Fig. 4(a), the molecule is much closer to the vacancy than in the planar case. From there, the water molecule has to overcome higher barriers to reach the intermediate stable states, since the system is less reactive, and most importantly the molecule cannot spontaneously dissociate over three "dangling bonds" (as in the planar geometry for IM4 and $K$ states).

However, if the oxygen atom saturates the $\mathrm{C}_{3}$ " dangling bond" first, as in the straight dissociation in graphene, the energetics for production of hydrogen are much more favorable. The direct dissociation proceeds with a low energy barrier of about $18 \mathrm{kcal} / \mathrm{mol}$.

To illustrate the reaction mechanism for the direct decomposition reaction we show in Fig. 4 the transition state geometry $[N-T S T$, Fig. 4(c)] and the final stable state with oxygen bonded to the $\mathrm{C}_{3}$ atom $[N-F$, Fig. 4(d)] while leaving the fivefold ring untouched. This configuration is not the only final state that the system can reach. An analogue of the $F$ state, namely, with the $\mathrm{O}$ atom in the middle of the vacancy site, can be reached through path- 
ways that involve intermediate configurations. In these other reaction paths, first one hydrogen atom saturates the $\mathrm{C}_{3}$ "dangling bond," and then the $\mathrm{OH}$ group completes the $s p^{3}$ carbon bonding leading to the exact analogue of the planar $I M 2$ state [N-IM2 in Fig. 4(b)]. The energy barrier for this reaction is about $20 \mathrm{kcal} / \mathrm{mol}$. Contrary to the graphene case this first configuration is the precursor of all the other possible intermediate states. The reconstructed bond in the fivefold ring has to be broken to reach, for instance, the $N-K$ and $N-I M 4$ states. This subsequent activation barrier is of the order of $\sim 25 \mathrm{kcal} / \mathrm{mol}$. From there, the dissociation reaction proceeds along similar lines as in the planar case. On the other hand, production of hydrogen from the $N-I M 2$ state is quite unfavorable $\left(E_{A} \sim\right.$ $70 \mathrm{kcal} / \mathrm{mol})$. A final comment on the analogue of the $E$ state, $N-E$ : even though it is still the global minimum of the PES, as in the planar case, now the reaction barrier for the water splitting from this state is substantially lower, about $46 \mathrm{kcal} / \mathrm{mole}$ versus $94 \mathrm{kcal} / \mathrm{mole}$ in graphene. It is thus clear that curvature can improve the energetics for the water dissociation reaction over a vacancy and drastically enhance the selectivity of the $\mathrm{H}_{2}$ production process.

For a quantitative assessment of the efficiency of the reaction, we estimated the equilibrium yield for the different cases of direct thermolysis, dissociation on a vacancy in graphene and in the nanotube, using the calculated PES and the vibrational frequencies of the relevant configurations. From the data displayed in Fig. 1 it is clear that the defective carbon substrate improves dramatically the equilibrium yield of $\mathrm{H}_{2}$ with respect to the dissociation in free space. This means that defects in graphitic materials would catalyze the dissociation of water with at least a factor of 2 gain in temperature with respect to the direct thermolysis that becomes competitive only well above $2000 \mathrm{~K}$.

Finally, we would like to point out that this reaction mechanism, as presented, is a batch process: once the vacancies in the carbon structure get saturated with oxygen, the process stops. It is then necessary to take the oxygen out to regenerate the active sites to make this a true catalytic process. There are several possibilities to achieve this, such as photoexcitation, or further reaction with other molecules. If such a process could be found, the energy needed to create the defects in the first place would be a one-time expenditure, and the system would thereafter work as a molecular energy conversion cycle: a true nanoscale chemical reactor.

The authors would like to acknowledge G. Parsons, T. Pearl, and H. Bock for stimulating discussions. This work has been supported in part by: ACS-PRF; NSF (DMR0304299 and CTS-0211792); BES, U.S. DOE at ORNL (DE-FG02-98ER14847 and DE-AC05-00OR22725 with UT-Battelle, LLC). Calculations have been carried out at CCS-ORNL, NCSU-HPC and San Diego and Pittsburgh Supercomputing Centers (NSF/NRAC NPA205). The molecular graphics in this Letter were done with VMD [28].
*M. K. Kostov and E. E. Santiso contributed in equal part to the research presented in this Letter.

Corresponding author.

Email address: mbnardelli@ncsu.edu

[1] J. A. Turner, Science 305, 972 (2004).

[2] A. Kogan, International Journal of Hydrogen Energy 25, 1043 (2000).

[3] A. C. Dillon, K. M. Jones, T. A. Bekkedahl, C. H. Klang, D. S. Bethune, and M. J. Heben, Nature (London) 386, 377 (1997).

[4] L. Schlapbach and A. Züttel, Nature (London) 414, 353 (2001).

[5] A.C. Dillon and M. J. Heben, Appl. Phys. A 72, 133 (2001).

[6] R. G. Parr and W. Yang, Density-Functional Theory of Atoms and Molecules (Oxford University Press, New York, 1989).

[7] E. E. Santiso and K.E. Gubbins, Mol. Simul. 30, 699 (2004).

[8] A. Hashimoto et al., Nature (London) 430, 870 (2004).

[9] K. Nordlund, J. Keinonen, and T. Mattila, Phys. Rev. Lett. 77, 699 (1996).

[10] J. R. Hahn and H. Kang, Phys. Rev. B 60, 6007 (1999).

[11] R. G. Parr and W. Yang, Density-Functional Theory of Atoms and Molecules (Oxford University Press, New York, 1989).

[12] D. Vanderbilt, Phys. Rev. B 41, 7892 (1990).

[13] Computer code CPMD, Copyright IBM Corp. 1990-2003, Copyright MPI für Festkörperforschung Stuttgart 19972001, http://www.cpmd.org.

[14] S. Baroni, A. Dal Corso, S. de Gironcoli, and P. Giannozzi, computer code PWSCF, http://www.pwscf.org.

[15] A. D. Becke, Phys. Rev. A 38, 3098 (1988).

[16] C. Lee, W. Yang, and R. G. Parr, Phys. Rev. B 37, 785 (1988).

[17] J. P. Perdew, K. Burke, and M. Ernzerhof, Phys. Rev. Lett. 77, 3865 (1996).

[18] A. Laio and M. Parrinello, Proc. Natl. Acad. Sci. U.S.A. 99, 12562 (2002).

[19] M. Iannuzzi, A. Laio, and M. Parrinello, Phys. Rev. Lett. 90, 238302 (2003).

[20] G. Mills, H. Jónsson, and G. K. Schenter, Surf. Sci. 324, 305 (1995).

[21] H. Jónsson, G. Mills, and K. W. Jacobsen, in Classical and Quantum Dynamics in Condensed Phase Simulations, edited by B.J. Berne, G. Ciccotti, and D.F. Coker (World Scientific, Singapore, 1998), pp. 385-404.

[22] G. Henkelman, B. P. Uberuaga, and H. Jónsson, J. Chem. Phys. 113, 9901 (2000).

[23] A. Banerjee, N. Adams, J. Simons, and R. Shepard, J. Phys. Chem. 89, 52 (1985).

[24] A. A. El-Barbary, R. H. Telling, C. P. Ewels, M. I. Heggie, and P. R. Briddon, Phys. Rev. B 68, 144107 (2003).

[25] P. A. Thrower and R. M. Mayer, Phys. Status Solidi A 47, 11 (1978).

[26] National Institute of Standards and Technology (NIST) online database.

[27] D. Townsend et al., Science 306, 1158 (2004).

[28] W. Humphrey, A. Dalke, and K. Schulten, J. Mol. Graphics 14, 33 (1996). 